\title{
Highly efficient voltage control of spin and enhanced interfacial perpendicular magnetic anisotropy in iridium-doped $\mathrm{Fe} / \mathrm{MgO}$ magnetic tunnel junctions
}

\author{
Takayuki Nozaki ${ }^{1,9}$, Anna Kozioł-Rachwal ${ }^{1,2,9}$, Masahito Tsujikawa ${ }^{3,4,9}$, Yoichi Shiota ${ }^{1}$, Xiandong Xu ${ }^{5}$, \\ Tadakatsu Ohkubo ${ }^{5}$, Takuya Tsukahara ${ }^{6}$, Shinji Miwa ${ }^{6,7}$, Motohiro Suzuki ${ }^{8}$, Shingo Tamaru' ${ }^{1}$, Hitoshi Kubota ${ }^{1}$, \\ Akio Fukushima ${ }^{1}$, Kazuhiro Hono ${ }^{5}$, Masafumi Shirai ${ }^{3,4}$, Yoshishige Suzuki ${ }^{1,6,7}$ and Shinji Yuasa ${ }^{1}$
}

Voltage control of spin enables both a zero standby power and ultralow active power consumption in spintronic devices, such as magnetoresistive random-access memory devices. A practical approach to achieve voltage control is the electrical modulation of the spin-orbit interaction at the interface between $3 d$-transition-ferromagnetic-metal and dielectric layers in a magnetic tunnel junction (MTJ). However, we need to initiate a new guideline for materials design to improve both the voltage-controlled magnetic anisotropy (VCMA) and perpendicular magnetic anisotropy (PMA). Here we report that atomic-scale doping of iridium in an ultrathin Fe layer is highly effective to improving these properties in Fe/MgO-based MTJs. A large interfacial PMA energy, $K_{\mathrm{i}, 0}$, of up to $3.7 \mathrm{~mJ} \mathrm{~m}^{-2}$ was obtained, which was 1.8 times greater than that of the pure Fe/MgO interface. Moreover, iridium doping yielded a huge VCMA coefficient (up to $320 \mathrm{fJ} \mathrm{Vm}^{-1}$ ) as well as high-speed response. First-principles calculations revealed that Ir atoms dispersed within the Fe layer play a considerable role in enhancing $K_{i, 0}$ and the VCMA coefficient. These results demonstrate the efficacy of heavy-metal doping in ferromagnetic layers as an advanced approach to develop high-density voltage-driven spintronic devices.

NPG Asia Materials (2017) 9, e451; doi:10.1038/am.2017.204; published online 5 December 2017

\section{INTRODUCTION}

Spintronic devices, such as a magnetoresistive random-access memory device using a MgO-based magnetic tunnel junction (MTJ), ${ }^{1,2}$ are expected to reduce the standby power of future computing systems by utilizing the non-volatile feature of magnetism. However, one significant challenge emerges from reducing the energy for information writing: magnetization switching. This issue is caused by electriccurrent-based operations of spintronic devices using electric-currentinduced magnetic fields, spin-transfer torque (STT) and spin-orbit torque based on the spin Hall effect rather than the electric-field-based operations presently used for semiconductor devices. For example, recent developments of STT-magnetoresistive random access memory have achieved $\sim 100 \mathrm{fJ}$ per bit writing energies, ${ }^{3}$ which corresponds to $10^{7} k_{\mathrm{B}} T$, where $k_{\mathrm{B}}$ is the Boltzmann constant and $T$ is the temperature (assumed to be $300 \mathrm{~K}$ here). In contrast, the energy required for maintaining magnetic information, that is, thermal stability, is between $60 k_{\mathrm{B}} T$ and $100 k_{\mathrm{B}} T$. This large energy gap between data writing and retention, on the order of $10^{5}$, mainly originates the unwanted energy consumption arising from the Ohmic dissipation of electric-current flow. Overcoming this fundamental issue requires the development of electric field manipulation of magnetization.

Among various approaches to the electric field manipulation of magnetism, ${ }^{4}$ the voltage-controlled magnetic anisotropy (VCMA) effect in a $3 d$ transition ultrathin ferromagnetic metal layer has attracted much attention as a promising solution. ${ }^{5,6}$ The achievement of the VCMA effect in MgO-based MTJs ${ }^{7}$ and the demonstration of high-speed responses, such as voltage-induced ferromagnetic resonance (FMR) excitation, ${ }^{8,9}$ spin-wave excitation ${ }^{10}$ and dynamic magnetization switching, ${ }^{11-14}$ have brought great changes to research in this field. Modulations of the Curie temperature, ${ }^{15}$ domain wall propagation, ${ }^{16,17}$ interfacial Dzyaloshinskii-Moriya interaction ${ }^{18}$ and proximity-induced magnetism ${ }^{19}$ have also been demonstrated. These results indicate the feasibility of constructing voltage-driven spintronic devices, such as voltage-torque magnetoresistive random access memory devices. ${ }^{20}$

\footnotetext{
${ }^{1}$ Spintronics Research Center, National Institute of Advanced Industrial Science and Technology, Ibaraki, Japan; ${ }^{2}$ Faculty of Physics and Applied Computer Science, AGH University of Science and Technology, Kraków, Poland; ${ }^{3}$ Research Institute of Electrical Communication, Tohoku University, Miyagi, Japan; ${ }^{4}$ Center for Spintronics Research Network, Tohoku University, Miyagi, Japan; ${ }^{5}$ National Institute for Materials Science, Research Center for Magnetic and Spintronic Materials, Ibaraki, Japan; ${ }^{6}$ Graduate School of Engineering Science, Osaka University, Osaka, Japan; ${ }^{7}$ Center for Spintronics Research Network, Osaka University, Osaka, Japan and ${ }^{8}$ Japan Synchrotron Radiation Research Institute (JASRI), Hyogo, Japan

9These authors contributed equally to this work.

Correspondence: Dr T Nozaki, Spintronics Research Center, National Institute of Advanced Industrial Science and Technology, Umezono1-1-1, Tsukuba, Ibaraki 305-8568, Japan.

E-mail: nozaki-t@aist.go.jp

Received 28 June 2017; revised 26 September 2017; accepted 6 October 2017
} 
The VCMA effect is observed at the interface between an ultrathin ferromagnetic metal and dielectric layers. This effect can be induced by purely electronic effects and by chemical or mechanical effects. The physical mechanisms of the purely electronic VCMA effect have been interpreted as the modification of the electronic structure at the interface through charge accumulation/depletion, ${ }^{21-23}$ an electricfield-induced magnetic dipole ${ }^{24}$ or the Rashba effect. ${ }^{25}$ Chemical effects, such as a voltage-induced redox reaction, ${ }^{26}$ or other electromigration ${ }^{27}$ or charge trapping ${ }^{28}$ effects can show a substantial VCMA coefficient of the order of a few thousands of $\mathrm{fJ} \mathrm{Vm}^{-1}$, which is defined as the induced surface anisotropy energy change per unit electric field. However, these mechanisms have drawbacks of limited operational speed and writing endurance. In contrast, for purely electronic VCMA effects, high-speed response with a sub-nanosecond timescale have already been demonstrated. However, the currently achieved amplitude of the VCMA coefficient of the purely electronic effect with high-speed response is insufficient $\left(\sim 100 \mathrm{fJ} \mathrm{Vm}^{-1}\right){ }^{29,30}$ For example, reversing the magnetization of a film with effective perpendicular magnetic anisotropy (PMA) energies of $0.2-0.5$ and $0.5-$ $2.0 \mathrm{~mJ} \mathrm{~m}^{-2}$, which correspond to the targets for cache and mainmemory applications, requires VCMA coefficients exceeding 200$500 \mathrm{fJ} \mathrm{Vm}^{-1}$ and 500-2000 f $\mathrm{Vm}^{-1}$, respectively, assuming an applied electric field of $1 \mathrm{~V} \mathrm{~nm}^{-1}$ (see Supplementary Information S1). As the purely electronic VCMA effect originates from the modification of the electronic structure at the interface, as mentioned above, an enhancement in the interfacial magnetic anisotropy, $K_{\mathrm{i}, 0}$, can be a basic guideline to enhance both the PMA and VCMA effects.

First-principles calculations predict that the utilization of high spinorbit interactions in heavy metals will enhance these properties. ${ }^{23,31} \mathrm{~A}$ few experimental attempts of interface engineering by heavy-metal insertion at a CoFe-based film $/ \mathrm{MgO}$ interface have been reported; however, a large VCMA coefficient over $100 \mathrm{fJ} \mathrm{Vm}^{-1}$ with enhanced interfacial PMA has not yet been achieved. ${ }^{30,32,33}$ Iridium (Ir) is a promising candidate for this purpose, because it has a huge spin-orbit coupling constant that is more than 10 times larger than those of iron and cobalt. ${ }^{31}$ For example, high interfacial PMA with a positive $K_{\mathrm{i}, 0}$ value of $1.5 \mathrm{~mJ} \mathrm{~m}^{-2}$ has been reported for $\mathrm{Ir} / \mathrm{Co}(111)$ multilayers. ${ }^{34}$ However, it is not easy to directly apply this interface in a voltagedriven MgO MTJ, because the mismatched interface band between Co (111) and $\mathrm{MgO}(001)$ degrades the coherent tunneling properties. In this study, we demonstrate that the introduction of an Ir-doped ultrathin $\mathrm{Fe}(001)$ layer can be used as a novel approach to improve the interfacial PMA and VCMA effects in Fe/MgO MTJs.

\section{EXPERIMENTAL PROCEDURES}

Multilayered structures consisting of $\mathrm{MgO}$ seed $(3 \mathrm{~nm}) / \mathrm{Cr}$ buffer $(30 \mathrm{~nm}) /$ ultrathin $\mathrm{Fe}\left(t_{\mathrm{Fe}}\right) / \mathrm{Ir}$ doping layer $\left(t_{\mathrm{Ir}}\right) / \mathrm{MgO}(2.5 \mathrm{~nm}) /$ top electrodes were prepared on $\mathrm{MgO}(001)$ substrates by a combination of molecular beam epitaxy and sputtering techniques. The $\mathrm{MgO}(001)$ seed and $\mathrm{Cr}(001)$ buffer layers were deposited at $200{ }^{\circ} \mathrm{C}$, followed by annealing at $800^{\circ} \mathrm{C}$. The ultrathin $\mathrm{Fe}(001)$ layer was grown at $200{ }^{\circ} \mathrm{C}$ and annealed at $260^{\circ} \mathrm{C}$. After the substrate cooled, the Ir doping layer and $\mathrm{MgO}$ (001) barrier were deposited at room temperature, followed by annealing at $350^{\circ} \mathrm{C}$. The $\mathrm{MgO}$ surface was capped by a sputterdeposited $30 \mathrm{~nm}$-thick ITO electrode to evaluate the magnetic properties or by a $30 \mathrm{~nm}$-thick $\mathrm{Cr}$ for scanning transmission electron microscopy observation. For the VCMA experiments, a $10 \mathrm{~nm}$-thick Fe top electrode was deposited at $200{ }^{\circ} \mathrm{C}$, followed by capping with $\mathrm{Ta} / \mathrm{Ru}$ layers. The nominal $\mathrm{Fe}$ and $\mathrm{Ir}$ thicknesses were varied in the ranges of $0.5 \mathrm{~nm} \leq t_{\mathrm{Fe}} \leq 1.4 \mathrm{~nm}$ and $0 \leq t_{\mathrm{Ir}} \leq$ $0.15 \mathrm{~nm}$, respectively.

The MTJ devices were prepared by conventional optical lithography, ionmilling and lift-off processes. The cross-sectional area of the junction was $2 \times 6 \mu \mathrm{m}^{2}$. The resistance-area product of the MTJ was designed to be $\sim 600 \mathrm{k} \Omega \mu \mathrm{m}^{2}$. Because of the high sample resistance, the maximum current

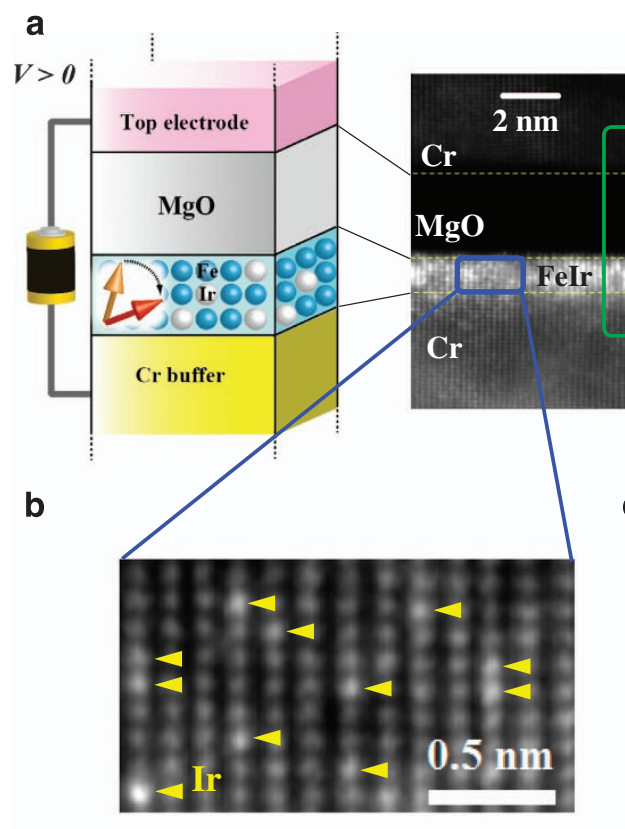

c
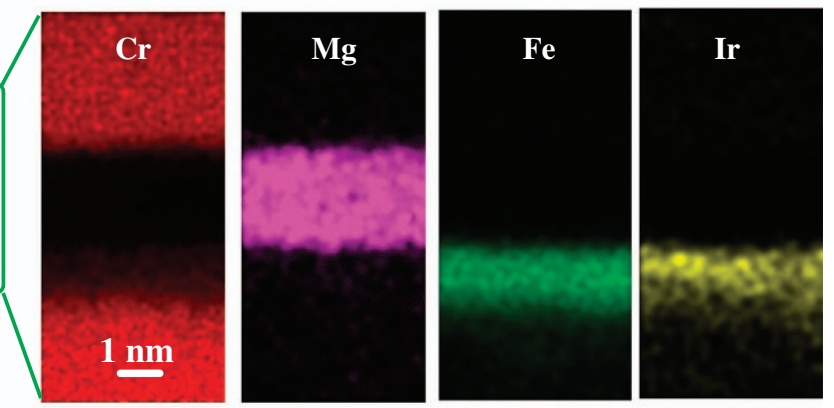

d

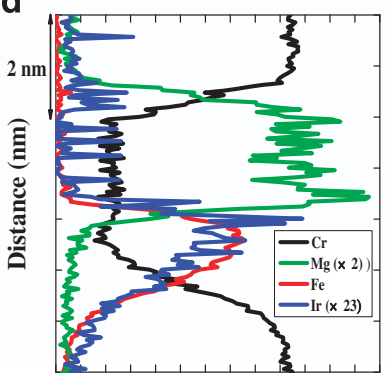

Count (a.u.) e

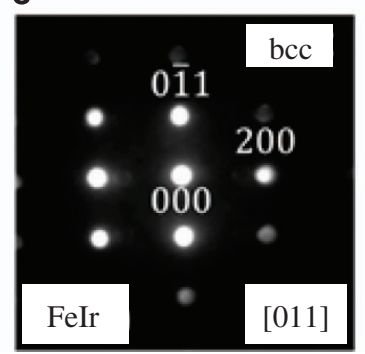

Figure 1 (a) Schematic illustration of the prepared junction structure. An Ir-doped ultrathin Fe layer was formed by interdiffusion under an annealing process from the Fe/lr bilayer structure (see details in the main text). (b) Atomic-scale Z-contrast high-angle annular dark-field scanning transmission electron microscopy (HAADF-STEM) imaging of the Ir-doped Fe layer. Substitutional doping of $\mathrm{Ir}$ in the bcc-Fe(001) layer is confirmed, as indicated by the yellow arrows. (c) Cross-sectional energy dispersive X-ray spectroscopy (EDS) maps and (d) intensity profiles of $\mathrm{Cr}, \mathrm{Mg}, \mathrm{Fe}$ and $\mathrm{Ir}$ elements for the $\mathrm{Cr}$ (30 nm)/Fe $(1.0 \mathrm{~nm}) / \mathrm{Ir}(0.05 \mathrm{~nm}) / \mathrm{MgO}(2.5 \mathrm{~nm}) / \mathrm{Cr}$ capping structure. (e) Nanobeam electron diffraction taken from the Ir-doped ultrathin Fe layer. 
a

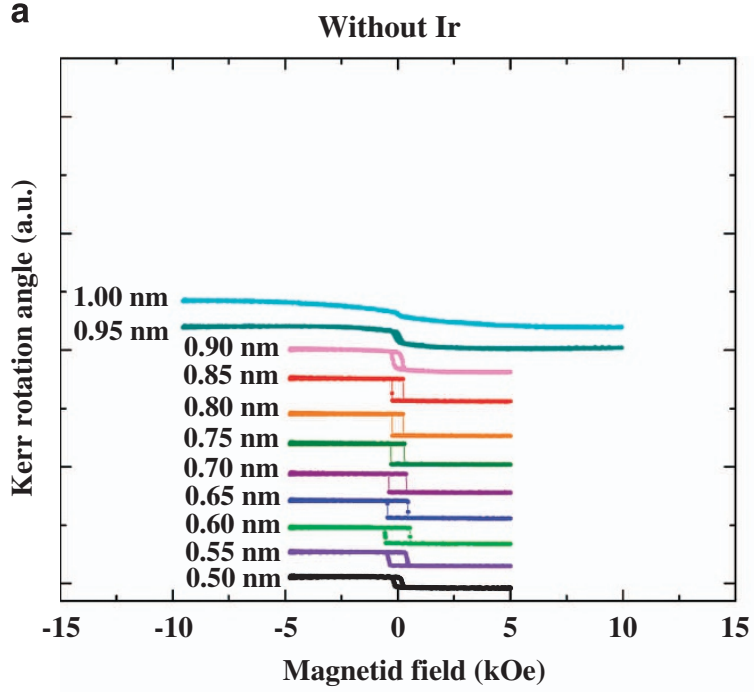

C

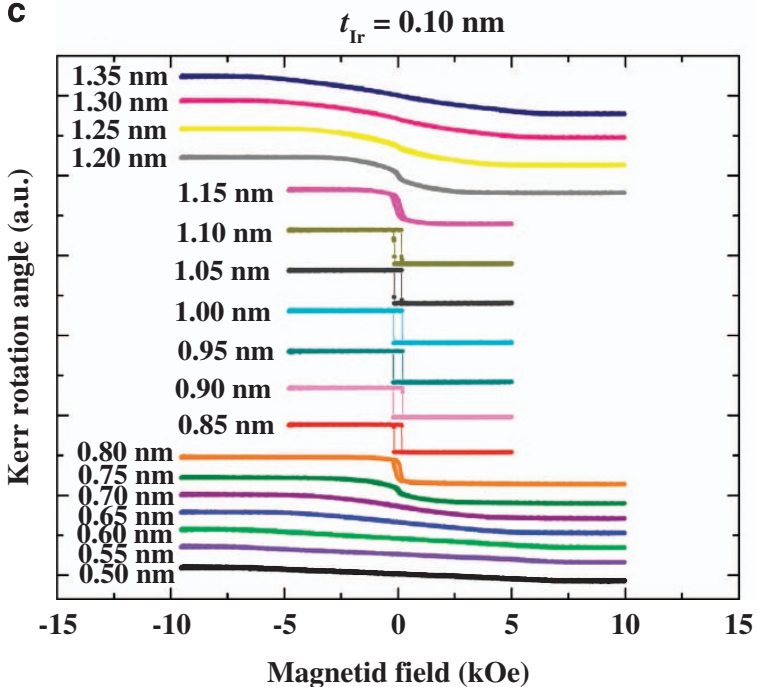

b

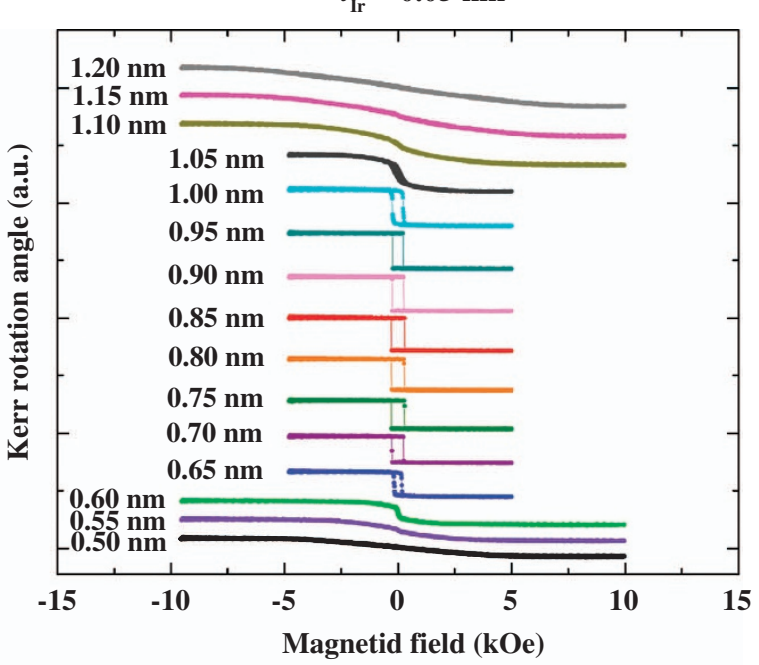

d

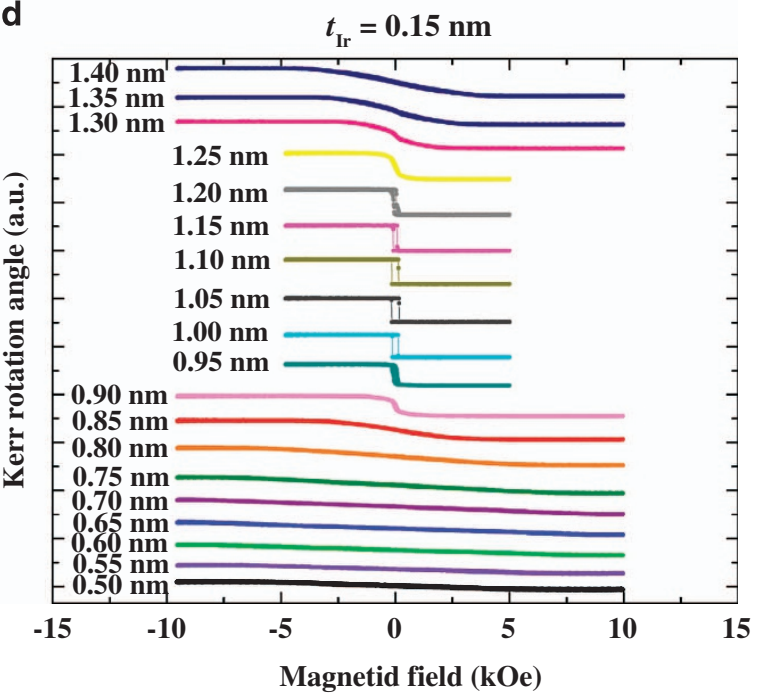

Figure 2 Fe thickness dependence of the polar-magneto-optic Kerr effect (MOKE) hysteresis curve for structures (a) without $\mathrm{Ir}$, (b) with $t_{\mathrm{lr}}=0.05 \mathrm{~nm}$, (c) with $t_{\mathrm{lr}}=0.10 \mathrm{~nm}$ and $(\mathbf{d})$ with $t_{\mathrm{lr}}=0.15 \mathrm{~nm}$.

density flowing in the MTJ under the maximum bias voltage application $( \pm 800 \mathrm{mV})$ was $\sim 1 \times 10^{2} \mathrm{~A} \mathrm{~cm}^{-2}$, which is small enough to exclude the influence of electric-current-induced torque, such as a current-induced magnetic field or STT.

\section{RESULTS AND DISCUSSION}

An ultrathin Ir doping layer was inserted between the $\mathrm{Fe}$ and $\mathrm{MgO}$ layers. However, we found that $\mathrm{Ir}$ atoms were dispersed into the $\mathrm{Fe}$ layer through an interdiffusion process during the post-annealing of the $\mathrm{MgO}$ layer. Figure $1 \mathrm{a}$ and $\mathrm{b}$ shows a schematic illustration of the prepared junctions and high-angle annular dark-field scanning transmission electron microscopy images of magnified area inside the Irdoped Fe layer for the sample with $t_{\mathrm{Fe}}=1.0 \mathrm{~nm}$ and $t_{\mathrm{Ir}}=0.05 \mathrm{~nm}$. Atomic-scale Z-contrast high-angle annular dark-field scanning transmission electron microscopy imaging enabled the identification of interdiffused Ir atoms as bright spots indicated by the yellow arrows. As seen in the energy dispersive X-ray spectroscopy elemental mappings for $\mathrm{Cr}, \mathrm{Mg}, \mathrm{Fe}$ and $\mathrm{Ir}$ shown in Figure 1c, the dispersion of Ir atoms into the ultrathin Fe layer is clearly confirmed. This result is also clarified in the intensity profile shown in Figure 1d, where the signal intensity for each element was adjusted to ensure the visibility of the shapes of the profiles. It is noteworthy that the shapes of the intensity profiles for $\mathrm{Fe}$ and $\mathrm{Ir}$ in Figure 1d are almost identical, suggesting the formation of a rather uniform FeIr solid solution. The high-angle annular dark-field scanning transmission electron microscopy image and nanobeam electron diffraction pattern in Figure 1e indicate the substitutional replacement of Fe by Ir atoms in bcc-Fe (see also Supplementary Information S2). The concentration of Ir is roughly estimated to be between 6 and $15 \%$ for $t_{\mathrm{Ir}}=0.05 \mathrm{~nm}$, and 12 and $21 \%$ for $t_{\mathrm{Ir}}=0.15 \mathrm{~nm}$.

The magnetic properties of the system were characterized by magneto-optic Kerr effect measurements. Figure 2 shows the magnetic hysteresis loops in the out-of-plane direction measured by polarmagneto-optic Kerr effect measurements as a function of $t_{\mathrm{Fe}}$ for different Ir doping layer thicknesses. As shown in Figure 2a, we noted PMA in the $t_{\mathrm{Fe}}$ range from 0.5 to $0.9 \mathrm{~nm}$ for $\mathrm{Cr} / \mathrm{Fe} / \mathrm{MgO}$; this result is attributed to $\mathrm{Fe} / \mathrm{MgO}$ interfacial anisotropy. ${ }^{35,36}$ The critical thickness of Fe, for which a spin reorientation transition (SRT) from the out-ofplane to in-plane direction occurs, was determined to be $0.9 \mathrm{~nm}$. The Ir doping in the ultrathin Fe layer has a distinct influence on the 


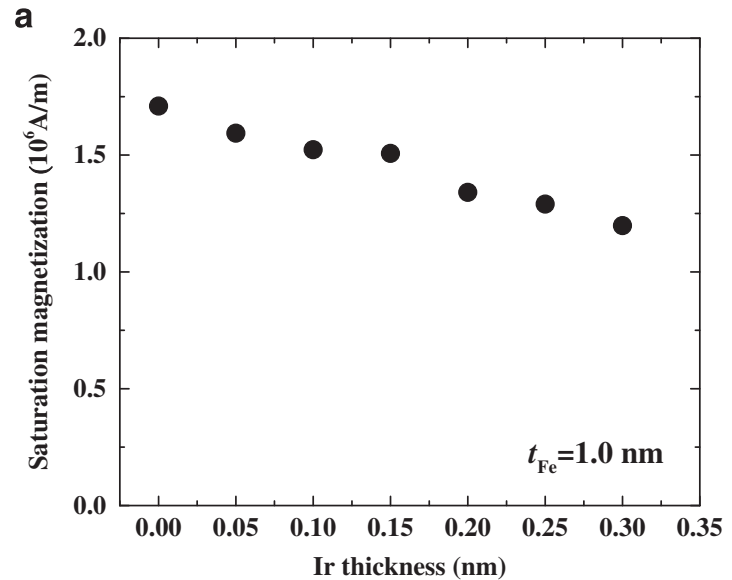

b

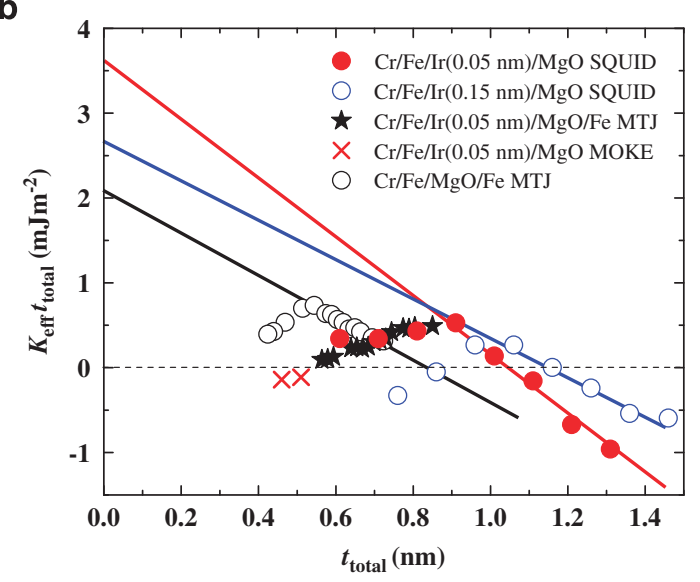

C

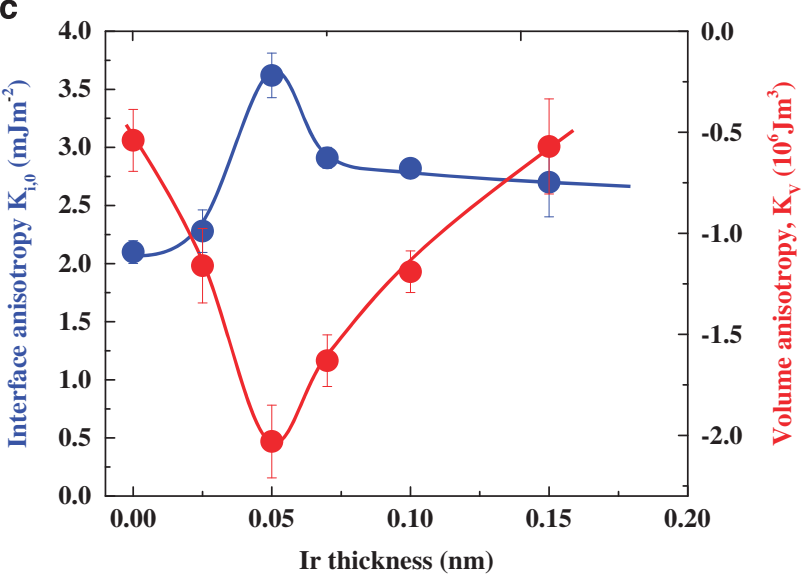

Figure 3 (a) Ir doping layer thickness, $t_{\mathrm{l}}$, dependence of the saturation magnetization values for a fixed $t_{\mathrm{Fe}}=1.0 \mathrm{~nm}$. (b) The $t_{\text {total }}$ dependences of $K_{\text {eff }} t_{\text {total }}$ for junctions with various Ir thicknesses. The colored circles represent the data obtained from the superconducting quantum interference device (SQUID) measurements. The red crosses represent the evaluated $K_{\text {eff }} t_{\text {total }}$ from the polar-MOKE measurements in the thinner Fe regime for $t_{1 \mathrm{r}}=0.05 \mathrm{~nm}$. The filled stars and open black circles exhibit the results obtained from the TMR measurements for the samples with $t_{1 \mathrm{r}}=0.05 \mathrm{~nm}$ and without the $\mathrm{Ir}$ doping layer, respectively. (c) The $t_{\mathrm{lr}}$ dependences of the interfacial magnetic anisotropy, $K_{\mathrm{i}, 0}$, and volume magnetic anisotropy, $K_{\mathrm{V}}$. The lines are guides to the eye.

magnetic properties of the system, as illustrated in Figure 2b-d. A key aspect of the observed tendency is that the out-of-plane easy axis becomes stable even in thicker Fe layers by Ir doping.
First, zero-remanence loops for the polar configuration were noted for very thin Fe layers, which indicates that in-plane anisotropy dominates for very thin Fe layers (see also Supplementary Information S3). After the appearance of full remanence in the perpendicular configuration, that is, the out-of-plane magnetic easy axis, an SRT from the out-of-plane to in-plane direction was observed. For the sample with Ir doping, two critical thicknesses can be defined: first, $t_{\mathrm{Fe}, \mathrm{SRT} 1}$, where the SRT from the in-plane to out-of-plane direction occurs with increasing $t_{\mathrm{Fe}}$ and a second, $t_{\mathrm{Fe}}$, SRT2, above which magnetization of the $\mathrm{Fe}$ layer is aligned along the easy $\mathrm{Fe}[001]$ inplane direction. The Fe thickness range for which the perpendicular magnetization direction occurs is shifted toward the thicker range when the Ir thickness is increased from $0.05 \mathrm{~nm}$ to $0.15 \mathrm{~nm}$. For example, while $t_{\mathrm{Fe}, \mathrm{SRT} 1}=0.6 \mathrm{~nm}$ and $t_{\mathrm{Fe}, \mathrm{SRT} 2}=1.05 \mathrm{~nm}$ were noted for $t_{\mathrm{Ir}}=0.05 \mathrm{~nm}$, and $t_{\mathrm{Fe}, \mathrm{SRT} 1}=0.9 \mathrm{~nm}$ and $t_{\mathrm{Fe}, \mathrm{SRT} 2}=1.25 \mathrm{~nm}$ were registered for $t_{\mathrm{Ir}}=0.15 \mathrm{~nm}$. These results show that the PMA effects for thicker Fe films were enhanced by Ir doping.

We examined the magnetic properties of the system quantitatively by performing complementary measurements using a superconducting quantum interference device magnetometer. The following discussion assumes an effective magnetic dead layer $\left(t_{\mathrm{d}}\right)$ with a thickness of $0.09 \mathrm{~nm}$, which can be estimated from the nominal Fe thickness dependence of the magnetic moment (see Supplementary Information S4). The existence of the $t_{\mathrm{d}}$ is mainly attributed to intermixing at the $\mathrm{Cr} / \mathrm{Fe}$ interface; ${ }^{36,37}$ we observed no clear changes in the $t_{\mathrm{d}}$ with changes in the inserted Ir thickness. Figure $3 \mathrm{a}$ shows the $t_{\mathrm{Ir}}$ dependence of the saturation magnetization $\left(M_{S}\right)$ for a fixed $t_{\mathrm{Fe}}=1.0 \mathrm{~nm}$. Here we assumed the total thickness of the free layer to be $t_{\text {total }}=t_{\mathrm{Fe}}+t_{\mathrm{Ir}}-t_{\mathrm{d}}$. Ir doping caused a monotonic reduction in the $M_{\mathrm{S}}$ value. We also confirmed the induced magnetic moment in Ir, whose spin moment couples in parallel with that of Fe, by X-ray absorption spectroscopy and XMCD measurements (see Supplementary Information S5).

Figure $3 \mathrm{~b}$ presents the $K_{\text {eff }} t_{\text {total }}$ dependences on $t_{\text {total }}$ obtained for $t_{\mathrm{Ir}}=0.05 \mathrm{~nm}$ (red dots) and $0.15 \mathrm{~nm}$ (blue open circles) as examples, where the effective anisotropy constants $\left(K_{\text {eff }}\right)$ were extracted from the superconducting quantum interference device measurements. For comparison, data for the $\mathrm{Cr} / \mathrm{Fe} / \mathrm{MgO}$ structure (open black circles) are also shown in the same graph. ${ }^{36}$ The $K_{\text {eff }} t_{\text {total }}$ values (red crosses) evaluated from the magneto-optic Kerr effect hysteresis loops in the thinner Fe ranges for $t_{\mathrm{Ir}}=0.05 \mathrm{~nm}$ are shown here as well. Positive $K_{\mathrm{eff}}$ $t_{\text {total }}$ values indicate the out-of-plane magnetic easy axis. The effective magnetic anisotropy energy (MAE) density, $K_{\text {eff, }}$ can be expressed by the phenomenological expression,

$$
K_{\text {eff }}=\left(K_{\mathrm{V}}-\frac{1}{2} \mu_{0} M_{\mathrm{S}}^{2}\right)+\frac{K_{i, 0}}{t_{\text {total }}}
$$

where $K_{\mathrm{V}}$ and $M_{\mathrm{S}}$ are the volume anisotropy and saturation magnetization, respectively. By plotting $K_{\text {eff }} t_{\text {total }}$ as a function of $t_{\text {total }}$, $K_{\mathrm{i}, 0}$ and $K_{\mathrm{V}}$ can be estimated from the $y$-intercept and slope, respectively. The Ir doping layer thickness dependences of $K_{\mathrm{i}, 0}$ and $K_{\mathrm{V}}$ are summarized in Figure $3 \mathrm{c}$. An extremely large $K_{\mathrm{i}, 0}$ value of $3.7 \pm 0.2 \mathrm{~mJ} \mathrm{~m}^{-2}$ is obtained for $t_{\mathrm{Ir}}=0.05 \mathrm{~nm}$. This value is $\sim 1.8$ times greater than that observed in the pure $/ \mathrm{MgO}$ interface $\left(2.0 \mathrm{~mJ} \mathrm{~m}^{-2}\right) .{ }^{35,36}$ Further doping of Ir leads to a reduction in this value; however, even at $t_{\mathrm{Ir}}=0.15 \mathrm{~nm}$, the $K_{\mathrm{i}, 0}$ value is still greater than that for the sample without Ir doping. It should be emphasized that no PMA was observed at the $\mathrm{Cr} / \mathrm{Ir}$-doped Fe interface (see Supplementary Information S6), similar to the $\mathrm{Cr} / \mathrm{Fe}$ structure. ${ }^{36}$ Therefore, the observed large $\mathrm{K}_{\mathrm{i}, 0}$ value mainly comes from the top Ir-doped $\mathrm{Fe} / \mathrm{MgO}$ single interface. 
a

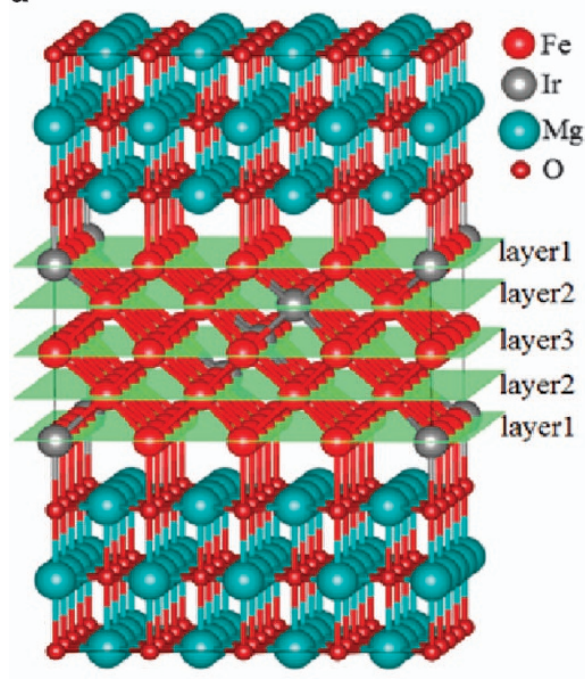

b

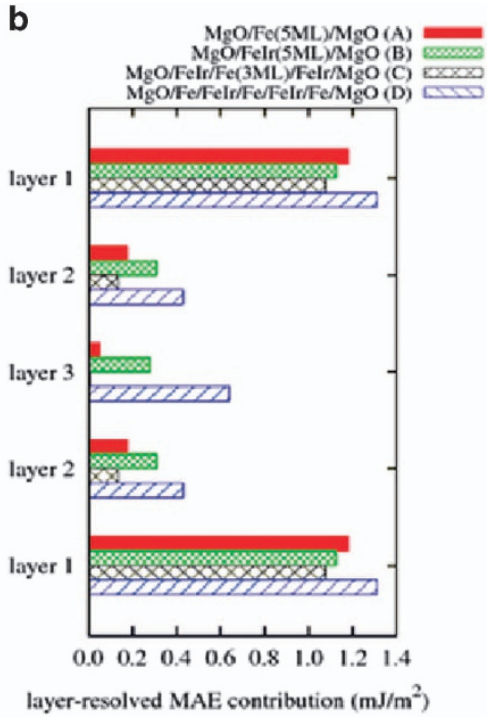

Figure 4 First-principles calculations of the magnetic anisotropy energy (MAE) in the Ir-doped Fe/MgO system. (a) Supercell structure used for the calculation of the $\mathrm{MgO}(5 \mathrm{ML}) / \mathrm{Fel}(5 \mathrm{ML}) / \mathrm{MgO}(5 \mathrm{ML})$ structure. (b) Layer-resolved MAE for Structure A (MgO/Fe(5ML)/MgO), Structure B (MgO/Felr(5ML)/MgO), Structure C (MgO/Felr(1ML)/Fe(3ML)/Felr(1ML)/MgO) and Structure D (MgO/Fe(1ML)/Felr (1ML)/Fe(1ML)/Felr(1ML)/Fe(1ML)/MgO). The Ir concentration was maintained at $6.25 \%$ in each Felr layer.

We performed first-principles density-functional calculations (see Supplementary Information S7 for details on the calculation conditions) to achieve a better understanding of the observed enhancement in $K_{\mathrm{i}, 0}$. Interfacial magnetic anisotropy in $\mathrm{Fe}(\mathrm{Co}) / \mathrm{Ir}$ multilayer structures has been discussed in previous works; ${ }^{31,38,39}$ however, the PMA induced by band hybridization among Fe, Ir and $\mathrm{MgO}$ should be very sensitive to the surrounding environment. Here we sought a more detailed comparison with the experimental results by considering the realistic structure of the Ir-doped ultrathin $\mathrm{Fe} / \mathrm{MgO}$ interface to shed light on the role of the interdiffused Ir atoms.

The basic structures used in calculations were Structure A $[\mathrm{MgO} / \mathrm{Fe} /$ $\mathrm{MgO}]$ and Structure B $[\mathrm{MgO} / \mathrm{FeIr} / \mathrm{MgO}]$, as shown in Figure $4 \mathrm{a}$ and Table 1, where the thickness of each layer was fixed to five monolayers (MLs). With this thickness of Fe, we observed a strong enhancement in PMA when $0.05 \mathrm{~nm}$-thick Ir was inserted at the $\mathrm{Fe} / \mathrm{MgO}$ interface. Thus, the Ir concentration in Structure B was fixed to 6.25\%. We also investigated the influence of the position of the Ir atoms on the MAE by considering the non-uniform dispersion of Ir in Structure C $[\mathrm{MgO} /$ $\operatorname{FeIr}(1 \mathrm{ML}) / \mathrm{Fe}(3 \mathrm{ML}) / \mathrm{FeIr}(1 \mathrm{ML}) / \mathrm{MgO}]$ and Structure $\mathrm{D}[\mathrm{MgO} / \mathrm{Fe}$ $(1 \mathrm{ML}) / \mathrm{Fe} \operatorname{Ir}(1 \mathrm{ML}) / \mathrm{Fe}(1 \mathrm{ML}) / \mathrm{Fe} \operatorname{Ir}(1 \mathrm{ML}) / \mathrm{Fe}(1 \mathrm{ML}) / \mathrm{MgO}$. The concentration of Ir was maintained at $6.25 \%$ for each FeIr layer in Structure $\mathrm{C}$ and D. Table 1 summarizes the calculated MAE for each structure. It is noteworthy that, in the calculations, two interfaces with $\mathrm{MgO}$ are assumed. Thus, the obtained value should be halved when a single interface is considered. The MAE for pure Fe/MgO (Structure A) was

Table 1 Magnetic anisotropy energies (MAEs) in the MgO/Fe(Ir)/MgO multilayer structures evaluated by first-principles calculations

\begin{tabular}{|c|c|c|}
\hline & Structure & $\operatorname{MAE}\left(m J m^{-2}\right)$ \\
\hline A & $\mathrm{MgO} / \mathrm{Fe} / \mathrm{Fe} / \mathrm{Fe} / \mathrm{Fe} / \mathrm{Fe} / \mathrm{MgO}$ & 3.13 \\
\hline B & MgO/Felr/Felr/Felr/Felr/Felr/MgO & 3.46 \\
\hline C & $\mathrm{MgO} / \mathrm{Fe} / \mathrm{r} / \mathrm{Fe} / \mathrm{Fe} / \mathrm{Fe} / \mathrm{Fe} / \mathrm{r} / \mathrm{MgO}$ & 2.66 \\
\hline D & $\mathrm{MgO} / \mathrm{Fe} / \mathrm{Fe} / \mathrm{r} / \mathrm{Fe} / \mathrm{Fe} / \mathrm{r} / \mathrm{Fe} / \mathrm{MgO}$ & 4.72 \\
\hline
\end{tabular}

The Ir concentration in each Felr layer was fixed at $6.25 \%$. estimated to be $3.13 \mathrm{~mJ} \mathrm{~m}^{-2}$. When Ir was dispersed uniformly within the Fe layer (Structure B), the MAE was increased to $3.46 \mathrm{~mJ} \mathrm{~m}^{-2}$. Interestingly, at the $\mathrm{MgO} / \mathrm{Fe} / \mathrm{FeIr}$ interface (Structure $\mathrm{D}$ ), we obtained a large enhancement in the MAE up to $4.72 \mathrm{~mJ} \mathrm{~m}^{-2}$, which is $\sim 1.5$ times greater than the result for the pure $\mathrm{Fe} / \mathrm{MgO}$ interface. Figure $4 \mathrm{~b}$ shows the layer-resolved MAE for each structure. It is noteworthy that the enhanced interfacial MAE can be attributed to the second and third layers, as well as the first interface layer adjacent to $\mathrm{MgO}$. In contrast, when $\mathrm{Ir}$ atoms concentrate at the interface with $\mathrm{MgO}$, the MAE decreases. This result is explained by the reduced interfacial MAE for Ir atoms $(0.16 \mathrm{meV}$ per atom) in comparison with that for $\mathrm{Fe}$ atoms $(0.60 \mathrm{meV}$ per atom). We also calculated the formation energy of an Ir-doped $\mathrm{Fe} / \mathrm{MgO}$ interface relative to bulk Ir and a nondoped $\mathrm{Fe} / \mathrm{MgO}$ interface. The formation energies for the $\mathrm{Fe} / \mathrm{MgO}$ interface with an FeIr layers inserted in the second and third layers were determined to be $72.8 \mathrm{meV}$ per Ir atom and $10.7 \mathrm{meV}$ per Ir atom, respectively. These values are much smaller than the results obtained for the Ir-doped-Fe/MgO interface (199 meV per Ir atom), suggesting that Ir prefers to diffuse into the Fe layer rather than to segregate to the $\mathrm{Fe} / \mathrm{MgO}$ interface. Therefore, the additional MAE from the Ir atoms interdiffused into the Fe layer plays an important role in enhancing $K_{\mathrm{i}, 0}$. Conventional ideas indicate that the interfacial PMA is dominated by the first mono-atomic layer at the interface; however, the present study suggests that material designs using heavymetal doping in the second and third layers offer new approaches to improving the interfacial PMA.

For the volume anisotropy term, $K_{\mathrm{V}}$, we observed a large negative value of $(-2.0 \pm 0.2) \times 10^{6} \mathrm{~J} \mathrm{~m}^{-3}$ for $t_{\mathrm{Ir}}=0.05 \mathrm{~nm}$. For this evaluation, a magnetostatic energy of $-1.6 \times 10^{6} \mathrm{~J} \mathrm{~m}^{-3}$ was considered from the $M_{\mathrm{S}}$ value. A possible contributor to the large negative $K_{\mathrm{V}}$ is lattice expansion induced by the Ir dispersion. The large negative value of $K_{\mathrm{V}}$ led to the total PMA undergoing a sharp reduction with an increasing $t_{\text {total }}$ despite a large $K_{\mathrm{i}, 0}$ value. Therefore, the suppression of the negative $K_{\mathrm{V}}$ is next important challenge to obtain a highly effective PMA energy at the target Fe thickness. 

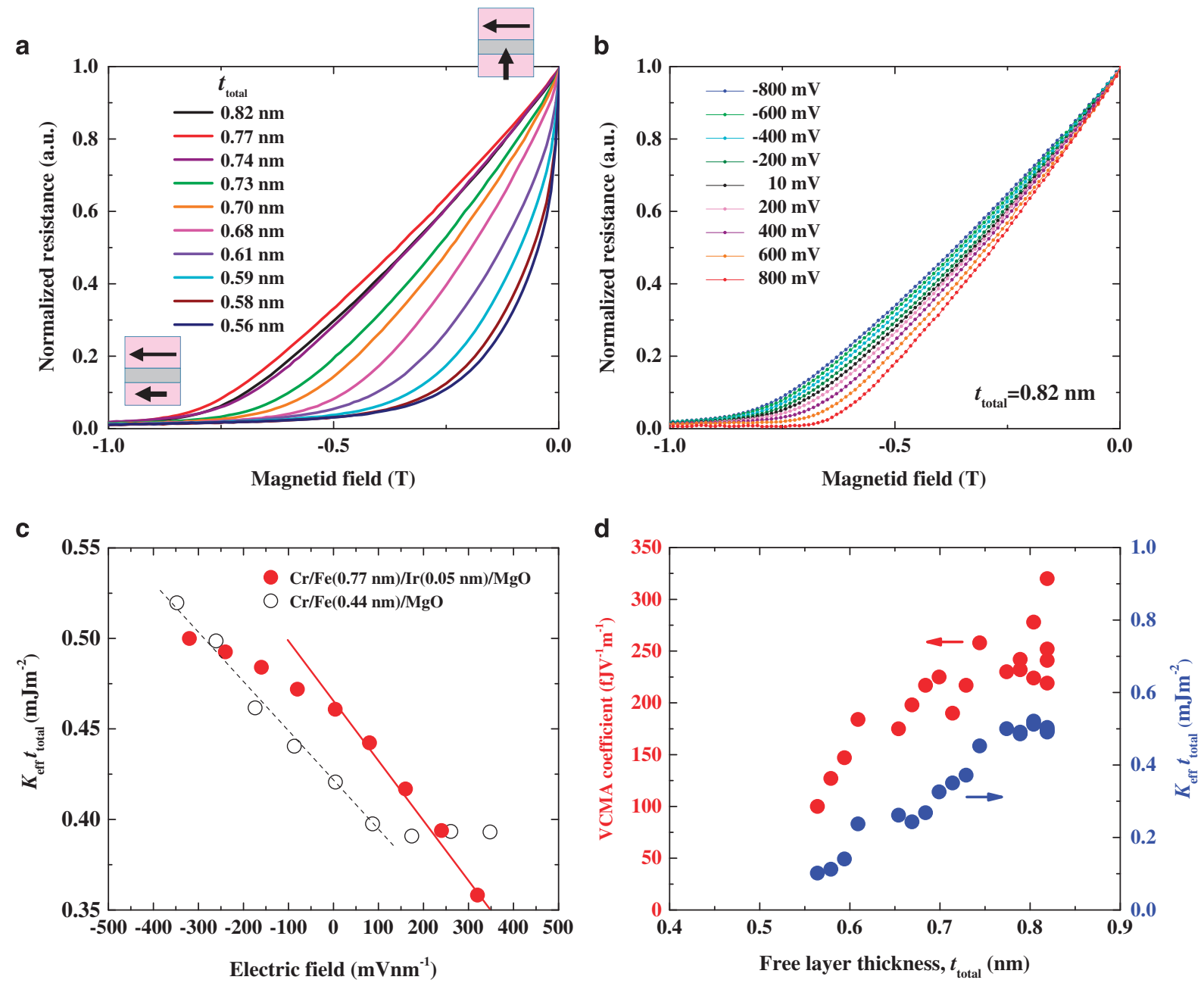

Figure 5 (a) Free-layer thickness, $t_{\text {total }}$, dependence of the normalized TMR curves measured under the application of an in-plane magnetic field. (b) Bias voltage dependence of the normalized TMR curves for the MTJ with $t_{\text {total }}=0.82 \mathrm{~nm}$. (c) Applied electric field dependence of $K_{\text {eff }} t_{\text {total }}$ observed for the Cr/lrdoped $\mathrm{Fe}(0.82 \mathrm{~nm}) / \mathrm{MgO}$ (red dots) and $\mathrm{Cr} / \mathrm{Fe}(0.44) / \mathrm{MgO}$ structures (open circles). (d) Free-layer thickness, $t_{\text {total }}$, dependence of the VCMA coefficient (red dots) evaluated from the slope in the positive electric field direction and $K_{\text {eff }} t_{\text {total }}$ values (blue dots).

We also observed a reduction in the PMA for thinner $t_{\text {total. }}$ One possible origin for this result is an increase of the Ir concentration close to the interface for thinner Fe layers, which should result in a reduced PMA according to first-principles calculations. We may also have encountered an influence of intermixing between Ir-doped Fe and $\mathrm{Cr}^{37}$ which can modify both the interface and volume contributions to the magnetic anisotropy in this system, as discussed with the $\mathrm{Cr} / \mathrm{Fe} / \mathrm{MgO}$ structure.

Next, VCMA experiments using the MTJ structures were performed. In these experiments, we focused on the sample with $t_{\mathrm{Ir}}=0.05 \mathrm{~nm}$, which showed the highest $K_{\mathrm{i}, 0}$ value. Here, $t_{\mathrm{Fe}}$ varied between 0.5 and $0.8 \mathrm{~nm}$, where the out-of-plane easy axis was firmly confirmed. The top $10 \mathrm{~nm}$-thick Fe layer acted as a reference layer with an in-plane magnetic easy axis. Two ferromagnetic layers assumed an orthogonal magnetization configuration under a zerostrength magnetic field. The application of an in-plane magnetic field, $H_{\mathrm{ex}}$, tilted the magnetization of the ultrathin Ir-doped Fe free layer to the in-plane direction. This behavior was observed as a gradual decrease in resistance through the tunneling magnetoresistance (TMR) effect, as illustrated by the drawings in Figure $5 \mathrm{a}$. The $t_{\text {total }}$ dependence of the TMR curves is also shown in Figure 5a. Here, the TMR curves were normalized by maximum $\left(H_{\mathrm{ex}}=0\right)$ and minimum $\left(H_{\mathrm{ex}}=-2 \mathrm{~T}\right)$ resistances to focus on the change in the saturation property. The saturation field, which reflects the effective PMA, increased with an increasing $t_{\text {total }}$. This trend is consistent with the results obtained by the superconducting quantum interference device measurements, as shown by the black stars in Figure 3b.

Figure $5 \mathrm{~b}$ displays an example of the normalized TMR curves $\left(t_{\text {total }}=0.82 \mathrm{~nm}\right)$ measured under application of bias voltages, $V_{\mathrm{b}}$, varying from $-0.8 \mathrm{~V}$ to $+0.8 \mathrm{~V}$. The polarity of $V_{\mathrm{b}}$ was defined relative to the top Fe layer. Clear shifts in the saturation field were observed depending on the amplitude of $V_{\mathrm{b}}$. The applications of positive and negative $V_{\mathrm{b}}$ values induced electron accumulation and depletion, respectively, at the $\mathrm{MgO}$ interface, causing decreases and increases in PMA, respectively. The observed sign was consistent with the conventional VCMA effect observed in Fe-based alloy/MgO junctions. ${ }^{4-6}$

As the tunneling conductance depends on the relative angle $(\theta)$ between two magnetic layers, the ratio of the in-plane component of the magnetization of the ultrathin Ir-doped Fe layer $\left(M_{\text {in-plane }}\right)$ to its 
saturation magnetization $\left(M_{\mathrm{S}}\right)$ can be calculated from the following relation: ${ }^{36}$

$$
\frac{M_{\text {in-plane }}}{M_{\mathrm{S}}}=\cos \theta=\frac{R_{90}-R(\theta)}{R(\theta)} \frac{R_{\mathrm{P}}}{R_{90}-R_{\mathrm{P}}}
$$

where $R_{\mathrm{P}}$ and $R_{90}$ are the MTJ resistances in the parallel and orthogonal configurations, respectively. Here, $R(\theta)$ is the MTJ resistance when the magnetization of the ultrathin Ir-doped Fe layer is tilted toward the in-plane direction at an angle $\theta$ from the film plane under an applied in-plane magnetic field. We calculated the effective PMA energy, $K_{\text {eff, }}$ under each $V_{\mathrm{b}}$ condition by combining the normalized $M-H$ curves with the $M_{\mathrm{S}}$ values obtained from the superconducting quantum interference device measurements.

Figure $5 c$ displays the applied electric field dependences of $K_{\text {eff }} t_{\text {total }}$ (red dots) for the MTJ with $t_{\text {total }}=0.82 \mathrm{~nm}$. Here, the applied electric field, $E$, defined as $E=V_{\mathrm{b}} / t_{\mathrm{MgO}}$, was varied from -320 to $+320 \mathrm{mV} \mathrm{nm}^{-1}$. The result of the largest VCMA effect observed in the $\mathrm{Cr} / \mathrm{Fe}\left(t_{\mathrm{Fe}}=0.44 \mathrm{~nm}\right) / \mathrm{MgO}$ structure is also shown in Figure $5 \mathrm{c}$ (open circles) for comparison. We found a small quadratic term in
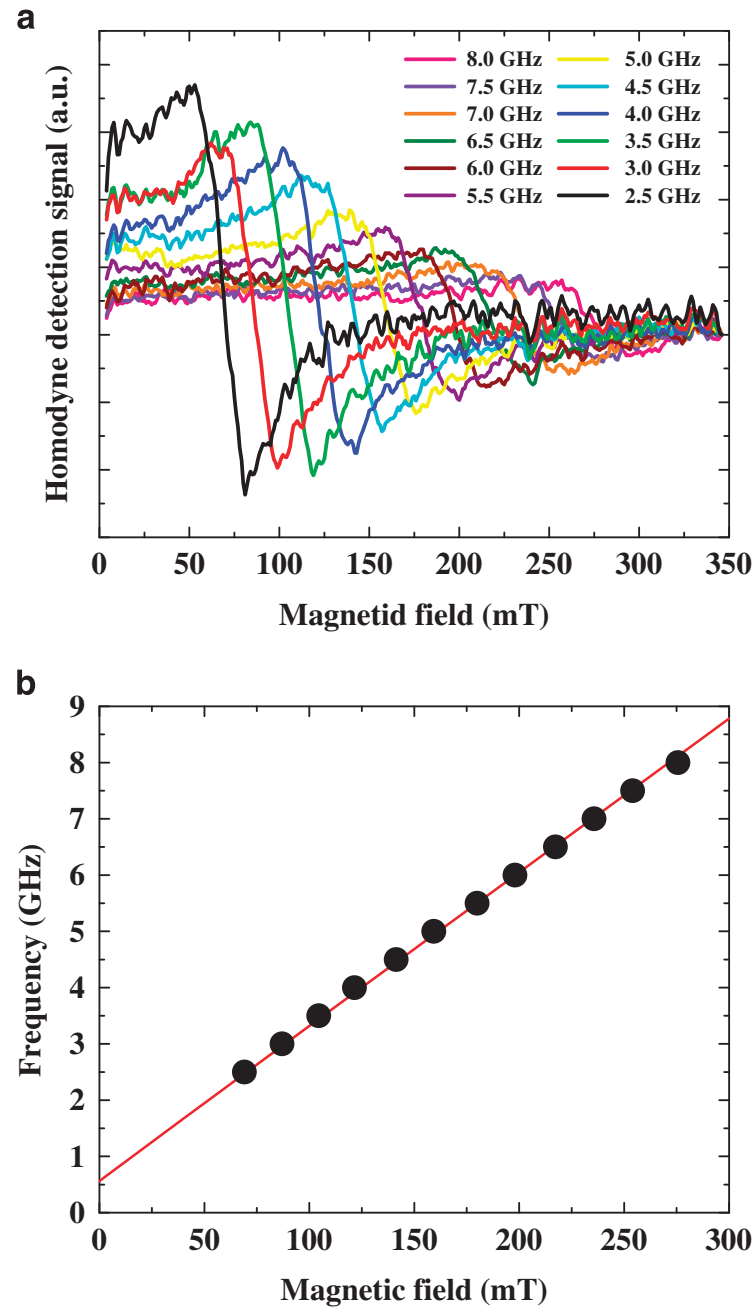

Figure 6 (a) External magnetic field dependence of the homodyne detection signal obtained under various applied radiofrequency (RF) power frequencies. The amplitude of the RF power and elevation angle, $\theta_{\mathrm{H}}$, of the external magnetic field were fixed at $-10 \mathrm{dBm}$ and $55^{\circ}$ from the in-plane direction, respectively. (b) Resonant frequency as a function of the magnetic field amplitude. The red line exhibits the fitting results based on the Kittel formula. addition to the dominant linear term for Ir-doped Fe. For the MTJs with a low resistance-area value, electric-current-induced heating or STT can cause quadratic behavior; however, this cause is not the case in our sample because of the sufficiently high resistance-area value. In addition, STT would prefer a parallel magnetization configuration under the negative bias voltage in our experimental configuration, which would cause a reduction in the saturation field. This is the opposite tendency observed in our experiment. Therefore, we can exclude influences from current-induced torque and the observed trend in the VCMA effect may relate to the intrinsic electronic structure at the Ir-doped $\mathrm{Fe} / \mathrm{MgO}$ interface.

To achieve voltage-induced dynamic magnetization switching of the perpendicularly magnetized free layer, ${ }^{11-14}$ we need to cancel the effective PMA during the switching process; therefore, the sign of the VCMA effect that induces the reduction in PMA is technologically important. In our previous study, we observed a large VCMA coefficient of $290 \mathrm{ff} \mathrm{Vm}^{-1}$ in the $\mathrm{Cr} / \mathrm{Fe} / \mathrm{MgO} \mathrm{MTJ}$, as shown by the open circles in Figure $5 c .{ }^{36}$ In that case, only an enhancement in PMA was obtained under the application of a negative bias voltage. In addition, high-speed operation was not fully confirmed. In contrast, the present study achieved a large reduction in PMA under positivebias voltage conditions. It is surprising that such a low concentration of Ir doping caused a drastic change in the VCMA property; this outcome suggests the extensive controllability of the VCMA effect by heavy-metal doping. From the linear fitting in the positive-bias direction, the VCMA coefficient was determined to be $320 \mathrm{fJ} \mathrm{Vm}{ }^{-1}$ for the MTJ with $t_{\text {total }}=0.82 \mathrm{~nm}$ (red line in Figure $5 \mathrm{c}$ ).

The dependence of the VCMA coefficient on the free-layer thickness $\left(t_{\text {total }}\right)$ in the positive-bias direction and the $K_{\text {eff }} t_{\text {total }}$ values are summarized in Figure 5d. A clear Fe thickness dependence of the VCMA coefficient was observed. The $K_{\text {eff }} t_{\text {total }}$ values also show a similar trend; the $K_{\text {eff }} t_{\text {total }}$ value increases with an increasing $t_{\text {total }}$. As the VCMA coefficient expresses the areal density of the anisotropy energy change induced per unit electric field, the coefficient is expected to be independent of the ferromagnetic layer thickness. This expectation arises from a simple consideration based on the carriermediated effect at the interface. However, the observed results imply that the VCMA coefficient is positively correlated with $K_{\text {eff }} t_{\text {total }}$, not simply with $K_{\mathrm{i}, 0}$. In such an ultrathin thickness regime, the electronic structure can possess a strong dependence on the thickness and can modify the VCMA effect. ${ }^{40}$ In addition, strain-induced modifications in the electronic structure can relate to the observed strong $\mathrm{Fe}$ thickness dependence; ${ }^{41}$ therefore, artificial precise control of these properties may also lead to a further enhancement in the VCMA properties. It should be emphasized that ensuring compatibility between a large VCMA coefficient and high effective PMA value is the key aspect for practical applications (see Supplementary Information S1).

The demonstration of high-speed response is important to show the practicality of the Ir-doped $\mathrm{Fe} / \mathrm{MgO}$ structure. For this purpose, we performed a voltage-induced FMR experiment using a homodyne detection method through the TMR effect (see Methods section and Nozaki et al. ${ }^{8}$ for details). Here, to excite the FMR dynamics effectively, we used the MTJ with $t_{\text {total }}=0.49 \mathrm{~nm}$, which is close to the critical thickness of the SRT $\left(t_{\mathrm{Fe}}, \mathrm{SRT} 1\right)$ for $t_{\mathrm{Ir}}=0.05 \mathrm{~nm}$. The amplitude of the voltage-induced FMR signal is proportional to $\cos \theta_{\mathrm{M}}$ $\sin ^{2} \theta_{\mathrm{M}}$, where $\theta_{\mathrm{M}}$ is the elevation angle of the magnetization from the film plane. ${ }^{8}$ This function has a maximum value at $\theta_{\mathrm{M}}=55^{\circ}$. Near the $t_{\mathrm{Fe}}$, SRT1, the magnetization can be easily directed along the external magnetic field due to the small effective anisotropy field. Then, we applied an external magnetic field with an elevation angle $\theta_{\mathrm{H}}=55^{\circ}$ to 

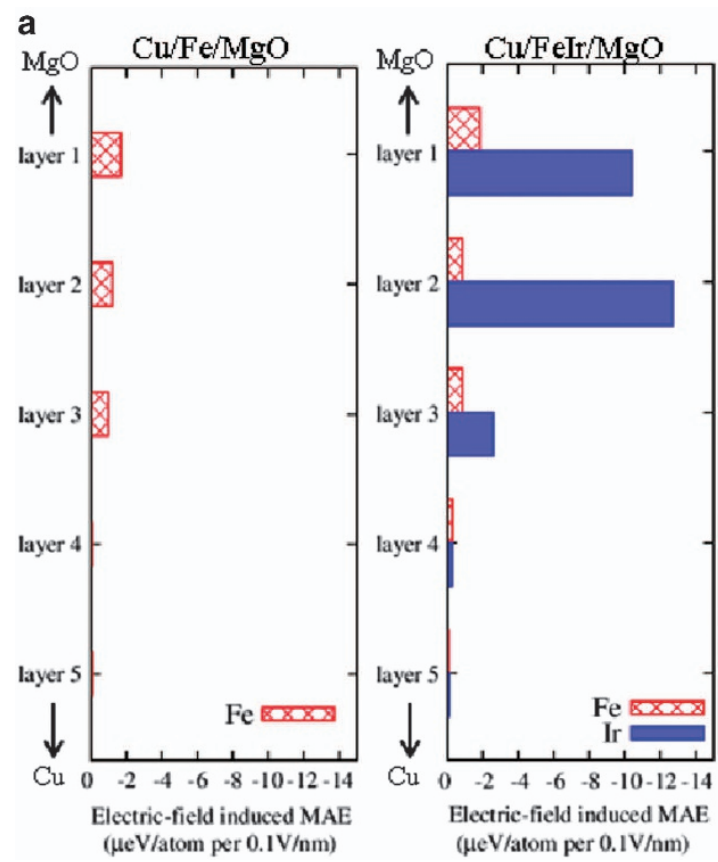

b
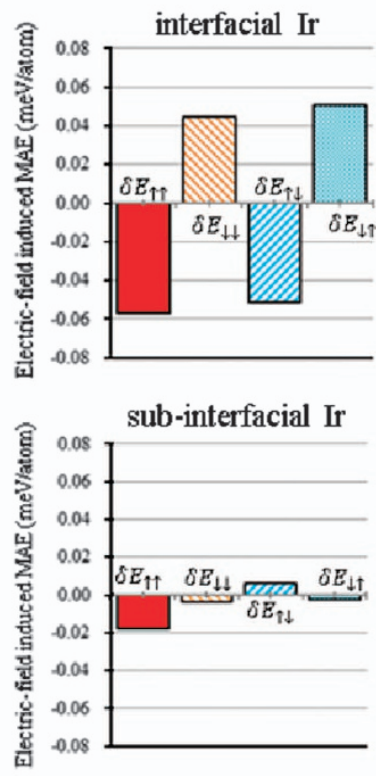

Figure 7 First-principles calculations of the electric field modulation on the MAE in the Ir-doped Fe/MgO system. (a) Atomic-resolved MAE change induced by an electric field of $0.1 \mathrm{~V} \mathrm{~nm}^{-1}$ in $\mathrm{MgO}$ for the $\mathrm{Cu} / \mathrm{Fe} / \mathrm{MgO}$ (left) and $\mathrm{Cu} / \mathrm{Fe} / \mathrm{r} / \mathrm{MgO}$ (right) structures. The Ir concentration was maintained at $6.25 \%$ in the Felr layer. The average value of 15 atoms in each layer is displayed for Fe. (b) Electric-field-induced MAE arising from the second-order perturbation of the spin-orbit coupling for the interfacial (top) and sub-interfacial (bottom) Ir atoms in the Cu/Felr/MgO structure.

maximize the output signal. The in-plane component of the $H_{\mathrm{ex}}$ was fixed parallel to the magnetic easy axis of the reference Fe layer. Under the application of radiofrequency power $(-10 \mathrm{dBm})$ with a fixed frequency, the amplitude of the external magnetic field was swept in the range of $0 \leq \mu_{0} H_{\mathrm{ex}} \leq 350 \mathrm{mT}$. As shown in Figure $6 \mathrm{a}$, we observed clear FMR signals with an anti-Lorentzian structure, indicating a VCMA-induced change in the effective anisotropy field in the measured magnetization configuration. ${ }^{8}$ Figure $6 \mathrm{~b}$ shows the applied magnetic field dependence of the resonant frequency. The resonant condition of the magnetization was fit by the conventional Kittel formula:

$$
\begin{aligned}
& 2 \pi f=-\gamma \sqrt{H_{1} H_{2}} \\
& H_{1}=H_{\mathrm{ex}} \cos \left(\theta_{\mathrm{H}}-\theta_{\mathrm{M}}\right)-H_{\mathrm{PMA}, \text { eff }} \sin ^{2} \theta_{\mathrm{M}} \\
& H_{2}=H_{\mathrm{ex}} \cos \left(\theta_{\mathrm{H}}-\theta_{\mathrm{M}}\right)+H_{\mathrm{PMA}, \text { eff }} \cos 2 \theta_{\mathrm{M}}
\end{aligned}
$$

where $\gamma$ is the gyromagnetic ratio, $\theta_{\mathrm{M}}$ is the angle of the magnetization direction of the ultrathin Fe layer from the in-plane axis and $H_{\mathrm{PMA} \text {,eff }}$ is the effective PMA field. The observed results are well reproduced with a fitting parameter of $H_{\mathrm{PMA}, \mathrm{eff}}=46 \mathrm{mT}$.

Although the VCMA coefficient and $K_{\text {eff }} t_{\text {total }}$ values show $t_{\text {total }}$ dependences, as discussed above, their monotonic trend should basically imply that the physical origin of the VCMA effect does not change with the $t_{\text {total }}$. One remaining concern is an influence of the voltage-induced modification of the Curie temperature for the thinnest Ir-doped $\mathrm{Fe}$ regime, for example, as observed in welldesigned few-ML Co. ${ }^{15}$ However, this effect gives a considerable influence only when the measurement temperature is close to the Curie temperature. On the other hand, we observed no drastic reduction in the magnetic moment near room temperature for the Ir-doped Fe layer (see Supplementary Information S8), suggesting that the VCMA effect is a predominately electronic origin and that its highspeed response should be maintained in the entire Ir-doped Fe thickness region. A voltage-induced FMR signal was not detected for the sample with $t_{\text {total }}=0.82 \mathrm{~nm}$. As we need a high external magnetic field to tilt the magnetization to surpass the high effective PMA, the precession of the voltage-induced FMR dynamics becomes smaller. Therefore, the direct demonstration of voltage-induced dynamic switching in the perpendicularly magnetized MTJ is required in the future.

To discuss the physical origin of the enhancement in the VCMA coefficient by Ir doping, we performed additional first-principles theoretical analysis of the VCMA effect in $\mathrm{Cu}(5 \mathrm{ML}) / \mathrm{Fe}(5 \mathrm{ML}) / \mathrm{MgO}$ (5ML) and $\mathrm{Cu}(5 \mathrm{ML}) / \mathrm{Fe} \operatorname{Ir}(5 \mathrm{ML}) / \mathrm{MgO}(5 \mathrm{ML})$ structures. Here, the Ir concentration was again fixed at $6.25 \%$. The MAE modulation of the electric field in $\mathrm{MgO}$ is enhanced from 94 to $127 \mathrm{fJ} \mathrm{Vm}^{-1}$ by $\mathrm{Ir}$ doping. Here, a dielectric constant of 10 was assumed for $\mathrm{MgO}$. The atomic-resolved electric-field-induced MAE for each structure is shown in Figure 7a. The electric-field-induced MAE for the Fe atoms are almost the same values between $\mathrm{Fe} / \mathrm{MgO}$ with and without Ir doping. On the other hand, the MAE modulation for the Ir atoms is more than five times greater than that for the Fe atoms at the FeIr/ $\mathrm{MgO}$ interface. To reveal the origin of huge VCMA effect for the Ir atoms, we calculated the MAE arising from a second-order perturbation of the spin-orbit coupling and divided the MAE contribution into spin-flip and spin-conserving process terms between the occupied and unoccupied states. ${ }^{42}$ Figure $7 \mathrm{~b}$ shows the voltage-induced MAE change arising from the second-order perturbation for the interfacial and subinterfacial Ir sites. The electric field modulation of the spin-conserving term between the majority spin occupied and unoccupied states $\left(\delta \mathrm{E}_{\uparrow \uparrow}\right)$ is larger than that between the minority spin states $\left(\delta \mathrm{E}_{\downarrow \downarrow}\right)$, as the majority spin $5 d$ states are dominant near the Fermi level in the interfacial and sub-interfacial Ir atoms (see Supplementary Information S7). On the other hand, the VCMA effect arising from the spin-flip term is very small, as the electric field modulation of the spin-flip terms $\left(\delta \mathrm{E}_{\uparrow \downarrow}\right.$ and $\delta \mathrm{E}_{\downarrow \uparrow}$ ) are almost the same value but opposite sign. We also estimated the electric-field-induced orbital 
magnetic moment anisotropy $\left(\Delta m_{L, S}=m_{L, S}^{\perp}-m_{L, S}^{/ /}\right)$of the Ir atoms, where $m_{L, S}^{\perp}(/ /)$ is the orbital magnetic moment in the out-of-plane (inplane) direction for the spin state $S(\uparrow$ or $\downarrow)$. The MAE is proportional to the $-\Delta m_{L, \uparrow}$ and $\Delta m_{L, \downarrow}$, then the spin-flip term $\left(\mathrm{E}_{\uparrow \downarrow}, \mathrm{E}_{\downarrow \uparrow}\right)$ is negligibly small. ${ }^{43}$ The $\Delta m_{L, S}$ values induced by an electric field of $0.1 \mathrm{~V} \mathrm{~nm}^{-1}$ are $\Delta m_{L, \uparrow}=0.00057$ and $\Delta m_{L, \downarrow}=0.00036$, and $\Delta m_{L, \uparrow}=0.00017$ and $\Delta m_{L, \downarrow}=-0.00003$ for the interfacial and sub-interfacial Ir atoms, respectively. The large modulation in $\Delta m_{L, \uparrow}$ also indicates that the electric-field-induced MAE is mainly caused by a modulation in the majority spin Ir- $5 d$ states.

We demonstrated a large VCMA coefficient of $320 \mathrm{fJ} \mathrm{Vm}^{-1}$ with a $K_{\text {eff }} t_{\text {total }}$ value of $\sim 0.46 \mathrm{~mJ} \mathrm{~m}^{-2}$ in the Ir-doped $\mathrm{Fe} / \mathrm{MgO}$ structure. The developed structure first achieved the required specifications for high-density voltage-torque magnetoresistive random access memory, corresponding to a technology node with a diameter of 25 to $30 \mathrm{~nm}$ for MTJ elements (see Supplementary Information S1). Firstprinciples calculations predict large enhancements in the interfacial PMA and VCMA effect in Fe/monolayer of heavy metal/MgO structures; ${ }^{31}$ however, the realization of uniform ML insertion is not easily achieved in practical MTJs. On the other hand, the Ir-doped Fe/ $\mathrm{MgO}$ structure should be much more manufacturable and have a high application possibility even for sputtering processes. We still have numerous choices for $4 d$ and $5 d$ elements, and therefore, materials engineering using heavy-metal doping has enormous possibilities for further improvements in interfacial PMA and VCMA properties.

\section{CONCLUSION}

We investigated the PMA and VCMA properties in Ir-doped Fe/MgO structures. A low concentration of Ir doping had a considerable influence on the magnetic properties and achieved a high interface magnetic anisotropy, $K_{\mathrm{i}, 0}$, up to $3.7 \mathrm{~mJ} \mathrm{~m}^{-2}$. In addition, a large VCMA coefficient of $320 \mathrm{fJ} \mathrm{Vm}{ }^{-1}$ was demonstrated in the $\mathrm{MgO}$ based MTJ. High-speed responsiveness was confirmed through voltage-induced FMR excitation. First-principles calculations revealed that Ir atoms dispersed within the ultrathin Fe layer had an important role to enhancing the $K_{\mathrm{i}, 0}$ and VCMA coefficient. Materials research using heavy-metal doping in $\mathrm{Fe} / \mathrm{MgO}$ junctions is a novel approach to improve the interfacial PMA and VCMA properties and promote the development of future spintronic devices with ultralow energy consumption.

\section{CONFLICT OF INTEREST}

The authors declare no conflict of interest.

\section{ACKNOWLEDGEMENTS}

We thank K Nakamura, T Oda, H Imamura, Y Miura, T Taniguchi, T Yorozu, Y Kotani, T Nakamura and R Jansen for fruitful discussions, and E. Usuda for assistance with experiments. This work was supported by the ImPACT Program of Council for Science. The X-ray absorption spectroscopy and XMCD measurements were performed at SPring- 8 with the approval of the Japan Synchrotron Radiation Research Institute (Proposal Number 2016B1017).

\section{PUBLISHER'S NOTE}

Springer Nature remains neutral with regard to jurisdictional claims in published maps and institutional affiliations.

1 Yuasa, S., Nagahama, T., Fukushima, A., Suzuki, Y. \& Ando, K. Giant roomtemperature magnetoresistance in single-crystal $\mathrm{Fe} / \mathrm{MgO} / \mathrm{Fe}$ magnetic tunnel junctions. Nat. Mater. 3, 868-871 (2004).
2 Parkin, S. S., Kaiser, C., Panchula, A., Rice, P. M., Hughes, B., Samant, M \& Yang, S.H. Giant tunnelling magnetoresistance at room temperature with MgO (100) tunnel barriers. Nat. Mater. 3, 862-867 (2004).

3 Ando, K., Fujita, S., Ito, J., Yuasa, S., Suzuki, Y., Nakatani, Y., Miyazaki, T. \& Yoda, H. Spin-transfer torque magnetoresistive random-access memory technologies for normally off computing (invited). J. Appl. Phys. 115, 172607 (2014).

4 Matsukura, F., Tokura, Y. \& Ohno, H. Control of magnetism by electric fields. Nat. Nanotechnol. 10, 209-220 (2015).

5 Weisheit, M., Fahler, S., Marty, A., Souche, Y., Poinsignon, C. \& Givord, D. Electric field-induced modification of magnetism in thin-film ferromagnets. Science $\mathbf{3 1 5}$, 349-351 (2007).

6 Maruyama, T., Shiota, Y., Nozaki, T., Ohta, K., Toda, N., Mizuguchi, M., Tulapurkar, A.A., Shinjo, T., Shiraishi, M., Mizukami, S., Ando, Y. \& Suzuki, Y. Large voltage-induced magnetic anisotropy change in a few atomic layers of iron. Nat. Nanotechnol. 4, 158-161 (2009).

7 Nozaki, T., Shiota, Y., Shiraishi, M., Shinjo, T. \& Suzuki, Y. Voltage-induced perpendicular magnetic anisotropy change in magnetic tunnel junctions. Appl. Phys. Lett. 96, 022506 (2010).

8 Nozaki, T., Shiota, Y., Miwa, S., Murakami, S., Bonell, F., Ishibashi, S., Kubota, H., Yakushiji, K., Saruya, T., Fukushima, A., Yuasa, S., Shinjo, T. \& Suzuki, Y. Electricfield-induced ferromagnetic resonance excitation in an ultrathin ferromagnetic metal layer. Nat. Phys. 8, 492-497 (2012).

9 Chen, Y.-J., Lee, H. K., Verba, R., Katine, J. A., Barsukov, I., Tiberkevich, V., Xiao, J. Q., Slavin, A. N., Krivorotov, I. N. Parametric Resonance of Magnetization Excited by Electrid Field. Nano Lett. 17, 572-577 (2017).

10 Verba, R., Carpentieri, M., Finocchio, G., Tiberkevich, V. \& Slavin, A. Excitation of propagating spin waves in ferromagnetic nanowires by microwave voltage-controlled magnetic anisotropy. Sci. Rep. 6, 25018 (2016).

11 Shiota, Y., Nozaki, T., Bonell, F., Murakami, S., Shinjo, T. \& Suzuki, Y. Induction of coherent magnetization switching in a few atomic layers of FeCo using voltage pulses. Nat. Mater. 11, 39-43 (2012).

12 Kanai, S., Yamanouchi, M., Ikeda, S., Nakatani, Y., Matsukura, F. \& Ohno, H. Electric field-induced magnetization reversal in a perpendicular-anisotropy CoFeB-MgO magnetic tunnel junction. Appl. Phys. Lett. 101, 122403 (2012).

13 Shiota, Y., Nozaki, T., Tamaru, S., Yakushiji, K., Kubota, H., Fukushima, A., Yuasa, S. \& Suzuki, Y. Evaluation of write error rate for voltage-driven dynamic magnetization switching in magnetic tunnel junctions with perpendicular magnetization. Appl. Phys. Express 9, 013001 (2016).

14 Grezes, C., Rojas Rozas, A., Ebrahimi, F., Alzate, J. G., Cai, X., Katine, J. A., Langer, J., Ocker, B., Khalili Amiri, P. \& Wang, K. L. In-plane magnetic field effect on switching voltage and thermal stability in electric-field-controlled perpendicular magnetic tunnel junctions. AIP Adv. 6, 075014 (2016).

15 Chiba, D., Fukami, S., Shimamura, K., Ishiwata, N., Kobayashi, K. \& Ono, T. Electrical control of the ferromagnetic phase transition in cobalt at room temperature. Nat. Mater. 10, 853-856 (2011).

16 Chiba, D., Kawaguchi, M., Fukami, S., Ishiwata, N., Shimamura, K., Kobayashi, K. \& Ono, T. Electric-field control of magnetic domain-wall velocity in ultrathin cobalt with perpendicular magnetization. Nat. Commun. 3, 888 (2012).

17 Schellekens, A. J., van den Brink, A., Franken, J. H., Swagten, H. J. \& Koopmans, B. Electric-field control of domain wall motion in perpendicularly magnetized materials. Nat. Commun. 3, 847 (2012).

18 Nawaoka, K., Miwa, S., Shiota, Y., Mizuochi, N. \& Suzuki, Y. Voltage induction of interfacial Dzyaloshinskii-Moriya interaction in Au/Fe/MgO artificial multilayer. Appl. Phys. Express 8, 063004 (2015).

19 Hibino, Y., Koyama, T., Obinata, A., Miwa, K., Ono, S. \& Chiba, D. Electric field modulation of magnetic anisotropy in perpendicularly magnetized $\mathrm{Pt} / \mathrm{Co}$ structure with a Pd top layer. Appl. Phys. Express 8, 113002 (2015).

20 Khalili Amiri, P., Alzate, J. G., Cai, X. Q., Ebrahimi, F., Hu, Q., Wong, K., Grezes, C. Lee, H., Yu, G., Li, X., Akyol, M., Shao, Q., Katine, J. A., Langer, J., Ocker, B. \& Wang, K. L. Electric-field-controlled magnetoelectric RAM: progress, challenges, and scaling. IEEE Transact. Magn. 51, 1-7 (2015).

21 Duan, C. G., Velev, J. P., Sabirianov, R. F., Zhu, Z., Chu, J., Jaswal, S. S. \& Tsymbal, E. Y. Surface magnetoelectric effect in ferromagnetic metal films. Phys. Rev. Lett. 101, 137201 (2008).

22 Nakamura, K., Shimabukuro, R., Fujiwara, Y., Akiyama, T., Ito, T. \& Freeman, A. J. Giant modification of the magnetocrystalline anisotropy in transition-metal monolayers by an external electric field. Phys. Rev. Lett. 102, 187201 (2009).

23 Tsujikawa, M. \& Oda, T. Finite electric field effects in the large perpendicular magnetic anisotropy surface Pt/Fe/Pt(001): a first-principles study. Phys. Rev. Lett. 102 247203 (2009).

24 Miwa, S., Suzuki, M., Tsujikawa, M., Matsuda, K., Nozaki, T., Tanaka, K., Tsukahara, T., Nawaoka, K., Goto, M., Kotani, Y., Ohkubo, T., Bonell, F., Tamura, E., Hono, K. Nakamura, T., Shirai, M., Yuasa, S. \& Suzuki, Y. Voltage controlled interfacial magnetism through platinum orbits. Nat. Commun. 8, 15848 (2017).

25 Barnes, S. E., leda, J. \& Maekawa, S. Rashba spin-orbit anisotropy and the electric field control of magnetism. Sci. Rep. 4, 4105 (2014).

26 Bauer, U., Yao, L., Tan, A. J., Agrawal, P., Emori, S., Tuller, H. L., van Dijken, S. \& Beach, G.S. Magneto-ionic control of interfacial magnetism. Nat. Mater. 14, 174-181 (2015).

27 Rajanikanth, A., Hauet, T., Montaigne, F., Mangin, S. \& Andrieu, S. Magnetic anisotropy modified by electric field in $\mathrm{V} / \mathrm{Fe} / \mathrm{MgO}(001) / \mathrm{Fe}$ epitaxial magnetic tunnel junction. Appl. Phys. Lett. 103, 062402 (2013).

28 Bauer, U., Przybylski, M., Kirschner, J. \& Beach, G. S. Magnetoelectric charge trap memory. Nano Lett. 12, 1437-1442 (2012). 
29 Skowroński, W., Nozaki, T., Shiota, Y., Tamaru, S., Yakushiji, K., Kubota, H., Fukushima, A., Yuasa, S. \& Suzuki, Y. Perpendicular magnetic anisotropy of Ir/CoFeB/MgO trilayer system tuned by electric fields. Appl. Phys. Express 8, 053003 (2015)

30 Li, X., Fitzell, K., Wu, D., Karaba, C. T., Buditama, A., Yu, G., Wong, K. L., Altieri, N., Grezes, C., Kioussis, N., Tolbert, S., Zhang, Z., Chang, J. P., Khalili Amiri, P. \& Wang, K.L. Enhancement of voltage-controlled magnetic anisotropy through precise control of Mg insertion thickness at CoFeBIMgO interface. Appl. Phys. Lett. 110, 052401 (2017).

31 Nakamura, K., Nomura, T., Pradipto, A.M., Nawa, K., Akiyama, T. \& Ito, T. Effect of heavy-metal insertions at Fe/MgO interfaces on electric-field-induced modification of magnetocrystalline anisotropy. J. Magn. Magn. Mater. 429, 214-220 (2017).

32 Bonaedy, T., Choi, J. W., Jang, C., Min, B.-C. \& Chang, J. Enhancement of electricfield-induced change of magnetic anisotropy by interface engineering of $\mathrm{MgO}$ magnetic tunnel junctions. J. Phys. D Appl. Phys. 48, 225002 (2015).

33 Lin, W., Vernier, N., Agnus, G., Lei, N., Eimer, S. \& Ravelosona, D. Interfacial charge accumulation effect on magnetic domain wall nucleation and propagation in a Pt_Co_Pt_Al203 structure. cond-mat.mtrl-sci arXiv:1201.5917 (2012).

34 Yakushiji, K., Kubota, H., Fukushima, A. \& Yuasa, S. Perpendicular magnetic tunnel junction with enhanced anisotropy obtained by utilizing an Ir/Co interface. Appl. Phys. Express 9, 013003 (2016).

35 Koo, J. W., Mitani, S., Sasaki, T. T., Sukegawa, H., Wen, Z. C., Ohkubo, T., Niizeki, T., Inomata, K. \& Hono, K. Large perpendicular magnetic anisotropy at $\mathrm{Fe} / \mathrm{MgO}$ interface. Appl. Phys. Lett. 103, 192401 (2013).

36 Nozaki, T., Kozioł-Rachwał, A., Skowroński, W., Zayets, V., Shiota, Y., Tamaru, S. Kubota, H., Fukushima, A., Yuasa, S. \& Suzuki, Y. Large voltage-induced changes in the perpendicular magnetic anisotropy of an MgO-based tunnel junction with an ultrathin Fe layer. Phys. Rev. Appl. 5, 044006 (2016).

37 Koziol-Rachwal, A., Nozaki, T., Freindl, K., Korecki, J., Yuasa, S. \& Suzuki, Y Enhancement of perpendicular magnetic anisotropy and its electric field-induced change through interface engineering in $\mathrm{Cr} / \mathrm{Fe} / \mathrm{MgO}$. Sci. Rep. 7, 5993 (2017)
38 Odkhuu, D., Rhim, S. H., Park, N. \& Hong, S. C. Extremely large perpendicular magnetic anisotropy of an $\mathrm{Fe}(001)$ surface capped by5dtransition metal monolayers: a density functional study. Phys. Rev. B 88, 184405 (2013).

39 Miura, Y., Tsujikawa, M. \& Shirai, M. A first-principles study on magnetocrystalline anisotropy at interfaces of $\mathrm{Fe}$ with non-magnetic metals. J. Appl. Phys. 113, 233908 (2013).

40 Tsujikawa, M., Haraguchi, S. \& Oda, T. Effect of atomic monolayer insertions on electric-field-induced rotation of magnetic easy axis. J. Appl. Phys. 111, 083910 (2012).

41 Ong, P. V., Kioussis, N., Amiri, P. K. \& Wang, K. L. Electric-field-driven magnetization switching and nonlinear magnetoelasticity in $\mathrm{Au} / \mathrm{FeCo} / \mathrm{MgO}$ heterostructures. Sci. Rep. 6, 29815 (2016).

42 Miura, Y., Ozaki, S., Kuwahara, Y. Tsujikawa, M., Abe, K. \& Shirai, M. The origin of perpendicular magneto-crystalline anisotropy in L1(0)-FeNi under tetragonal distortion. J. Phys. Condens. Matter 25, 106005 (2013).

43 Bruno, P. Tight-binding approach to the orbital magnetic moment and magnetocrystalline anisotropy of transition-metal monolayers. Phys. Rev. B 39, 865 (1989).

This work is licensed under a Creative Commons Attribution 4.0 International License. The images or other third party material in this article are included in the article's Creative Commons license, unless indicated otherwise in the credit line; if the material is not included under the Creative Commons license, users will need to obtain permission from the license holder to reproduce the material. To view a copy of this license, visit http:// creativecommons.org/licenses/by/4.0/

(C) The Author(s) 2017

Supplementary Information accompanies the paper on the NPG Asia Materials website (http://www.nature.com/am) 